1 Title: Evaluating the Usability of a Smartphone Virtual Seating Coach Application for Powered

2 Wheelchair Users

4 Authors' Names \& Author's Affiliations:

5 Yu-Kuang Wu, MS PT ${ }^{1,2}$ (yuw39@pitt.edu), Hsin-Yi Liu, PhD PT ${ }^{1,2}$ (hsl16@pitt.edu), Annmarie

6 Kelleher, MS OTR/L ${ }^{1,2}$ (akellehe@pitt.edu), Jonathan Pearlman, PhD¹,2(ilp46@pitt.edu), Rory A.

7 Cooper, $\operatorname{PhD}^{1,2}$ (rcooper@pitt.edu)

$8{ }^{1}$ Human Engineering Research Laboratories, ${ }^{2}$ Veterans Affairs Pittsburgh Healthcare System,

9 Department of Rehabilitation Science and Technology

10 Address: 6425 Penn Avenue, Suite 400, Pittsburgh, PA 15206

11

12 Corresponding author:

13 Rory A. Cooper, PhD

14 Address: Human Engineering Research Laboratories, VA Pittsburgh Healthcare System, 6425

15 Penn Avenue, Suite 400, Pittsburgh, PA 15206;

16 Telephone number: 412-822-3700;

17 Fax: 412-822-3699

18 Email: rcooper@pitt.edu

20 Present/ permanent address:

21 Human Engineering Research Laboratories, VA Pittsburgh Healthcare System, 6425 Penn

22 Avenue, Suite 400, Pittsburgh, PA 15206 
2

\title{
Evaluating the Usability of a Smartphone Virtual Seating Coach Application for
} Powered Wheelchair Users

\author{
Yu-Kuang Wu, MS PT ${ }^{1,2}$, Hsin-Yi Liu, MS PT ${ }^{1,2}$, Annmarie Kelleher, MS OTR/L ${ }^{1,2}$, Jonathan Pearlman, \\ $\mathrm{PhD}^{1,2}$, Rory A. Cooper, $\mathrm{PhD}^{1,2}$ \\ ${ }^{1}$ Human Engineering Research Laboratories, Department of Veterans Affairs Pittsburgh \\ Healthcare System \\ Pittsburgh, PA, USA
}

${ }^{2}$ Department of Rehabilitation Sciences and Technology, School of Health and Rehabilitation Sciences, University of Pittsburgh

Abstract

The aim of the smartphone virtual seating coach (SVSC) was to provide a personalized reminder/warning system to encourage powered wheelchair users to use their powered seating functions (PSFs) as clinically recommended. This study evaluated the usability of the SVSC system by gathering feedback from five powered wheelchair users and five rehabilitation professionals through questionnaires and interviews. The results indicated that clear and understandable instructions to adjust the PSFs are the most important requirement for SVSC application. The instructions must be intuitive, could benefit from animations or indications of PSFs control buttons so powered wheelchair users can adjust their PSFs immediately and appropriately. Powered Seating Functions

Abbreviations:

SVSC Smartphone Virtual Seating Coach

PSFs Powered Seating Functions 
Powered seating functions (PSFs), which include tilt in space, backrest recline, and legrest

47 elevation, are critical functions for people who stay in their powered wheelchairs for long periods.[1] Through altering tilt and recline angles, positioning mechanisms help people with limited trunk control maintain seating stability and to re-position their body.[2] Adjusting posture dynamically and maintaining proper postural alignment by using PSFs is important for managing complications such as orthostatic hypotention, autonomic dysreflexia, spasticity, and edema.[1] frequent and well-performed repositioning exercises can redistribute skin pressure, thus reducing the risk of developing pressure ulcers.[3] Appropriate seating adjustments may also improve driving safety. For example, using the tilt function to compensate for the changes in the center of gravity while driving on a slope may prevent the wheelchair from tipping over or the user from sliding out of the wheelchair.[1, 4]

The Virtual Seating Coach (VSC) provides a personalized system which can promote proper utilization of PSFs based on clinical recommendations.[5, 6] By comparing the seating function usage data with the clinical recommendations, the system provides tailored reminders to the users.[5] The VSC includes safety warnings, such as tilt angle adjustments while driving on slopes and repositioning reminders. Clinicians can customize the coaching program based on individual conditions, hence training can be extended beyond the clinical setting. Through individualized reminders proper usage of powered seating functions can be facillitated in their daily environment.

The Smartphone VSC (SVSC) was designed as an easily installed add-on system to be applied to powered wheelchairs with PSFs. In SVSC, accelerometers were connected to a microcontroller which converted the analog sensor signals into digital data and sent them to an Android smartphone. [7] [8] A Kalman filter was applied to decrease the noise. A smartphone cradle was designed to be easily mounted on the armrest of powered wheelchairs. The cradle allowed the users to position the smartphone based on their individual preferences to view the information displayed on the smartphone and operate their smartphones with greater ease. Our SVSC app has the ability to analyze the seating angle data and give users recommendations. (Figure 1) For example, when users are driving downhill on a steep ramp, the 
69 app will remind the users to tilt the seat backward to improve sitting stability. The app shows the angle

70 information as well as the target angle so the users can understand how to adjust their seating angles. The

71 reminder display effects will change depending on how the users set up their smartphones' sound effects.

72 [9] The purpose of this study was to test the usability of and gather feedback about the hardware and

73 software designed for our SVSC system from powered wheelchair users and rehabilitation professionals.

Usability Study

76

77

78

79

80

81

82

83

84

85

86

87

88

89

90

91

\section{Participants}

The usability test of SVSC was performed with two groups of participants. One group was comprised of wheelchair users who had experience using PSFs. The other group consisted of rehabilitation professionals with experience in prescribing powered wheelchairs with powered seating functions, which included therapists, physicians, or engineers. According to a study that investigated the number of subjects needed for usability testing, 80 percent of usability problems can be detected by the first four or five subjects.[10] Therefore, five participants were recruited for each group in this study. The only criteria to recruit powered wheelchair users was to be 18 years or older and have the ability to operate a powered wheelchair equipped with powered seating functions as a primary means of mobility. All participants were required to have been using a smartphone more than one year so they understood how to operate most of the main functions of a smartphone, such as reading text messages, launching an app, etc. There were no exclusion criteria for either group of participants.

Protocol

This usability study was approved by the Institutional Review Board of the University of Pittsburgh. This study centered on the assessment of the usability of the SVSC via a questionnaire and an

\section{Abbreviations:}

SVSC Smartphone Virtual Seating Coach

PSFs Powered Seating Functions 
interview with open-ended questions. For the powered wheelchair users, the SVSC was installed on their personal powered wheelchair. The researcher gave a brief introduction of the SVSC system including the purpose of SVSC and an overview of the SVSC app. Participants were presented with two tasks: 1) receive a repositioning reminder and adjust the seating angle following the instructions delivered by SVSC and 2) receive a safety warning reminder and adjust the seating angle following the instructions delivered by SVSC. In order to understand the users' preferences for the display layout and effects, the repositioning and safety warning reminders were set differently. The repositioning reminder appeared on the top of the screen (Figure 1A) and showed three choices ("OK", "Snooze" and "Cancel") for users to decide if they wanted to perform the repositioning exercise. The safety warning reminders appeared as text messages (Figures 1B and 1C). To check a safety warning reminder, the users needed to scroll down the navigation bar and click the text message, which is the same way that most smartphone users check their incoming text messages. The feedback messages for all of the reminders and warnings included a short description about the reminder/warning and an angle bar indicating the recommended angle range of the powered seating functions for users to adjust. The reminders/warning will not disappear if the users do not follow the instruction to adjust their wheelchair, or follow the instruction incorrectly. After completing these two tasks, the participants were given sufficient time to use the app and to ask any questions about the SVSC system, and then were asked to fill out a questionnaire with a series of usability questions and were interviewed to gather their feedback and opinions about the SVSC system. The rehabilitation professionals were asked to complete the same two tasks as the powered wheelchair user group, and were also asked to review the function of customizing the coach app, including the adjustability of settings for safety warnings and repositioning exercise reminders. Afterward, they were asked to respond to the same questionnaire and were also interviewed. All powered wheelchair users were encouraged to participate in an in-home trial lasting up to five days. During the in-home trial, the SVSC system was on their powered wheelchair all the time so the users could experience how the SVSC worked in their daily lives. This could help determine if the SVSC would cause annoyance to the users or interfere with their daily 
117 activities. After this in-home trial, all the participants were asked to answer the same questionnaire and

118 interviewed to determine whether they had any additional feedback.

Questionnaire and Interview

A paper-based questionnaire was used to gather the participants' feedback, which collected

122 demographic information, and included 24 questions about general impressions of the SVSC system. Five

123 common usability $[9,11]$ components were included in the questionnaires: the visibility of the SVSC app;

124 ease of use of the app; error prevention; and usefulness and satisfaction in terms of hardware and

125 software.[12, 13] Users rated usability on a five point likert scale. Other questions asked which features of

126 the SVSC system the participants liked most, whether the participants would use or recommend it to their

127 friends to use if the system becomes commercial, and their impressions of the SVSC. Participants were

128 interviewed to provide an open forum for discussing concerns or to provide suggestions with respect to 129 the hardware and software of SVSC system.

\section{Data Analysis}

Principal component analysis (PCA) was used to test construct of usability questions. Descriptive

133 analysis was performed to compare the differences in responses between the two visits of the powered 134 wheelchair user group (PW visit 1 and PW visit 2) and rehabilitation professionals (Rehab Pro). The PC 135 algorithm was performed to infer causal links with acyclic directed graph among different usability 136 questions in the questionnaire from powered wheelchair users based on correlation test and the 137 Spearman's rho was used to perform the correlation test.[14]

\section{Abbreviations:}

SVSC Smartphone Virtual Seating Coach

PSFs Powered Seating Functions 
Construct validation of questionnaire

The scree plot of the PCA (Figure 2) shows the presence of five factors with Eigen values > 1,

142 representing $90 \%$ of total variance in the index. Furthermore, after analyzing each question in each factor,

143 most of the questions can be categorized in the five factors, which are visibility, ease of use, error

144 prevention, usefulness and satisfaction. As a result, our questionnaire has the ability to gather the usability 145 information from our participants in this study.

147 Visibility

Figure 3 shows the results about visibility from the powered wheelchair users and rehabilitation professionals. The powered wheelchair users and rehabilitation professionals in our study reported that the information was clearly delivered by the SVSC interface. The app provided statistical information for the compliance of performing the repositioning exercise and the number of safety warning reminders to 152 the users. However, the statistics display of PSFs usage (clear stats chart display) and safety warning reminders (clear safety warning reminder for seat functions) were two items with lower scores for powered wheelchair users after the in-home trial. Our SVSC app used charts to present statistical changes, but the small smartphone display seemed to be not able to deliver the information clearly. The second issue of visibility was the safety warning reminders, which appeared as text messages. Some participants

157 reported that the text message was too easy to ignore and they usually did not check the text messages. 158 The navigation bar sometimes contained several icons of other different smartphone apps, leading to the 159 fact that it would be harder to notice the reminders from the SVSC. For these reasons, having the reminders pop up on the screen would be a better way to remind powered wheelchair users to adjust 161 seating angles. The rehabilitation professionals remarked about the small font size in our SVSC app. It 162 was suggested that the SVSC should have font size options, or voice feedback messages/instructions for 163 people with poor visual perception. 
Ease of Use and Error Prevention

Figure 4 shows the results from the ease of use and error prevention analysis. The "easy to navigate the application" item received the lowest scores from both powered wheelchair users and rehabilitation professionals. Users were asked to perform different steps for repositioning reminders and safety warnings. For the safety warning reminders, the alert appeared as text messages. To check the

170 reminders and enter the instruction screen, the users needed to click the text messages from the navigation

171 bar. For the repositioning reminders, the alert came up on the screen, and the users could choose if they 172 wanted to perform the repositioning exercise. Results showed most participants preferred the direct on173 screen reminders; indicating the safety warning reminders required too many steps to access the 174 instructions. Rehabilitation professionals noted that users with impaired fine motor function, might have 175 challenges with accessing text messages. To reduce user error, the SVSC app automatically executes once 176 the USB connection is made. SVSC is powered from the charging socket of the powered wheelchair. If 177 the USB is not connected or the power jack is loose, the app will generate an error message indicating that 178 there is a problem with the power connection. In our study, most of the users liked this design.

Usefulness and Satisfaction

Figure 5 shows results for usefulness and satisfaction. The usefulness ratings of the SVSC 182 increased after the in-home trials for powered wheelchair users. Results indicate that intention to use the 183 SVSC decreased after the in-home trial, but the powered wheelchair users tended to recommend the 184 SVSC to other users. They reported already understanding the safety reminders and had a schedule to 185 perform repositioning exercises. Rehabilitation professionals indicated that they would recommend the 186 SVSC to their clients. The scores of the "sufficient functions and capabilities" item were low when 187 compared to other items. Results indicate that the system needs to include a desktop software element or

\section{Abbreviations:}

SVSC Smartphone Virtual Seating Coach

PSFs Powered Seating Functions 
web-based dashboard to present a summary of PSF usage to review usage reports and adjust SVSC settings. Table 2 and Table 3 show the features of the SVSC participants preferred most and their concerns about the hardware/software from the questionnaire.

The SVSC implements selected coaching algorithms to remind users to use their PSFs appropriately. These coaching reminders are important clinical recommendations to prevent ineffective usage behaviors which may decrease sitting stability, result in tipping incidents, or induce complications due to prolonged static sitting. In our current design, the safety warning reminders kept reminding the users until they adjusted their seating angles appropriately. However, these coaching reminders might not be ideal in some situations. For example, when users are on the wheelchair, the system would remind them to have some minimal tilt angle to increase sitting stability. However, this warning reminder becomes inappropriate when the user wants to get close to a dining table with the height that can only accommodate the sitting position without being tilted. Another dilemma is legrest usage. When users elevate their legrests to a relatively large angle, it is recommended that they need to recline their backrest backward to prevent the over stretched hamstring muscles from rotating their pelvis posteriorly, causing a slouched posture in the wheelchair. This reminder, however, might not be suitable when they need legrest clearance to drive through places with obstacles where the mild tilt angle is not enough. To avoid continuously reminding users under these situations, the safety warning reminders need to have the option which allows the users to choose whether or not they should follow the recommendation to adjust their wheelchair based on their current situation. Another way to deal with this issue is using context awareness technology to determine the appropriateness of sending reminders to users.[5]

\section{Causal Relationship from the Questionnaire}

The causal relationship discovered by the PC algorithm showed that the instructions were understood to adjust the powered seating functions in response to the safety warnings $\left(\mathrm{r}_{\mathrm{s}}=0.614, p=.02\right)$ and repositioning reminders $\left(\mathrm{r}_{\mathrm{s}}=0.624, p=.017\right)$ were causes for powered wheelchair users to 
recommend the application to other users. Participants would recommend the SVSC to other users if the

214 the instructions were very clear. In our current design, the SVSC provides an angle meter with target

215 angle. Powered wheelchair users expected the reminders to tell them specific PSF(s) they needed to adjust,

216 how they have to adjust them, and the reason that the PSFs need to be adjusted. Some users suggested that

217 the instructions include the button for the PSF on the controller or having the animations of changing

218 specific PSF.

Limitations

Conducting the usability test after presenting a working prototype to end users might limit some creative ideas from users about the application. Another limitation is that new powered wheelchair users

223 were not recruited in this usability test. New powered wheelchair users may provide a different perspective. Small sample size might be an issue for the data representation of the target population. However, the idea of the usability study is to gather feedback from the end users and refine the development. All powered wheelchair users in this study had already used smartphones for more than 4

227 years. Using accelerometers to measure angle was unstable $\left( \pm 3^{\circ}\right)$ while the wheelchair was moving, and the wires connecting the microcontroller to the accelerometers increased the width. The ideal design for the SVSC would minimize the wiring and/or use wireless data transmission. tests provide information necessary to refine the SVSC system. Future development should provide more intuitive instructions to adjust PSFs in the SVSC app, reduce size and wires, and have an interface for rehabilitation professionals to review and tune the SVSC app.

\section{Abbreviations:}

SVSC Smartphone Virtual Seating Coach

PSFs Powered Seating Functions 
Conflicts of Interest

238 The authors (Y Wu, H Lui, J Pearlman, RA Cooper) wish to disclose that they are the inventors of the

239 Seating Function Monitoring and Coaching System (US Patent Application 14/609, 747), which has been

240 licensed by the University of Pittsburgh to Permobil, USA as the Virtual Seating Coach by Permobil,

241 USA. The authors do not have any personal financial interest in Permobil, USA.

242

243 Funding

244 This research was completed with the support of the National Science Foundation (Grant \#EEC0540865 -

245 Quality of Life Technology Engineering Research Center), and the University of Pittsburgh.

246

247 Ethical Approval

248 Institutional Review Board of the University of Pittsburgh (PRO13010589).

249

250 References

251 1. Dicianno, B.E., et al., RESNA position on the application of tilt, recline, and elevating legrests for 252 wheelchairs. Assist Technol, 2009. 21(1): p. 13-22; quiz 24.

253 2. Lange, M., Positioning: it's all in the angles. Advance for Occupational Therapy Practitioners, 2542006

255 3. Henderson, J.L., et al., Efficacy of three measures to relieve pressure in seated persons with 256 spinal cord injury. Arch Phys Med Rehabil, 1994. 75(5): p. 535-9.

257 4. Corfman, T.A., et al., Tips and falls during electric-powered wheelchair driving: effects of seatbelt use, legrests, and driving speed. Arch Phys Med Rehabil, 2003. 84(12): p. 1797-802. 
5. Ding, D., et al., Virtual coach technology for supporting self-care. Phys Med Rehabil Clin N Am, 2010. 21(1): p. 179-94.

6. Liu, H.-Y., et al., Seating virtual coach: A smart reminder for power seat function usage. Technology and Disability, 2010. 22(1): p. 53-60.

7. Ding, D., et al., Usage of tilt-in-space, recline, and elevation seating functions in natural environment of wheelchair users. J Rehabil Res Dev, 2008. 45(7): p. 973-83.

8. Yu-Kuang Wu, Hsin-Yi Liu, Josh Brown, Annmarie Kelleher, Hongwu Wang, Rory A. Cooper. A Smartphone Application For Improving Powered Seat Functions Usage: A Preliminary Test. in Rehabilitation Engineering and Assistive Technology Society of North America Conference. 2013. Seattle, WA: RESNA.

9. Barnum, C.M., Usability Testing Essentials: Ready, Set...Test! 2010: Elsevier Science.

10. Virzi, R.A., Refining the test phase of usability evaluation: how many subjects is enough? Hum. Factors, 1992. 34(4): p. 457-468.

11. Jakob, N., Usability inspection methods, in Conference Companion on Human Factors in Computing Systems \%@ 0-89791-651-4.1994, ACM: Boston, Massachusetts, USA. p. 413-414.

12. Nielsen, J., Usability inspection methods, in Conference Companion on Human Factors in Computing Systems. 1994, ACM: Boston, Massachusetts, USA. p. 413-414.

13. Nielsen, J., Enhancing the explanatory power of usability heuristics, in Proceedings of the SIGCHI Conference on Human Factors in Computing Systems. 1994, ACM: Boston, Massachusetts, USA. p. 152-158.

14. Harris, N. and M. Drton, PC algorithm for nonparanormal graphical models. J. Mach. Learn. Res., 2013. 14(1): p. 3365-3383.

15. Walker, M., L. Takayama, and J.A. Landay. High-fidelity or low-fidelity, paper or computer? Choosing attributes when testing web prototypes. in Proceedings of the Human Factors and Ergonomics Society Annual Meeting. 2002. SAGE Publications.

Abbreviations:

SVSC Smartphone Virtual Seating Coach

PSFs Powered Seating Functions 

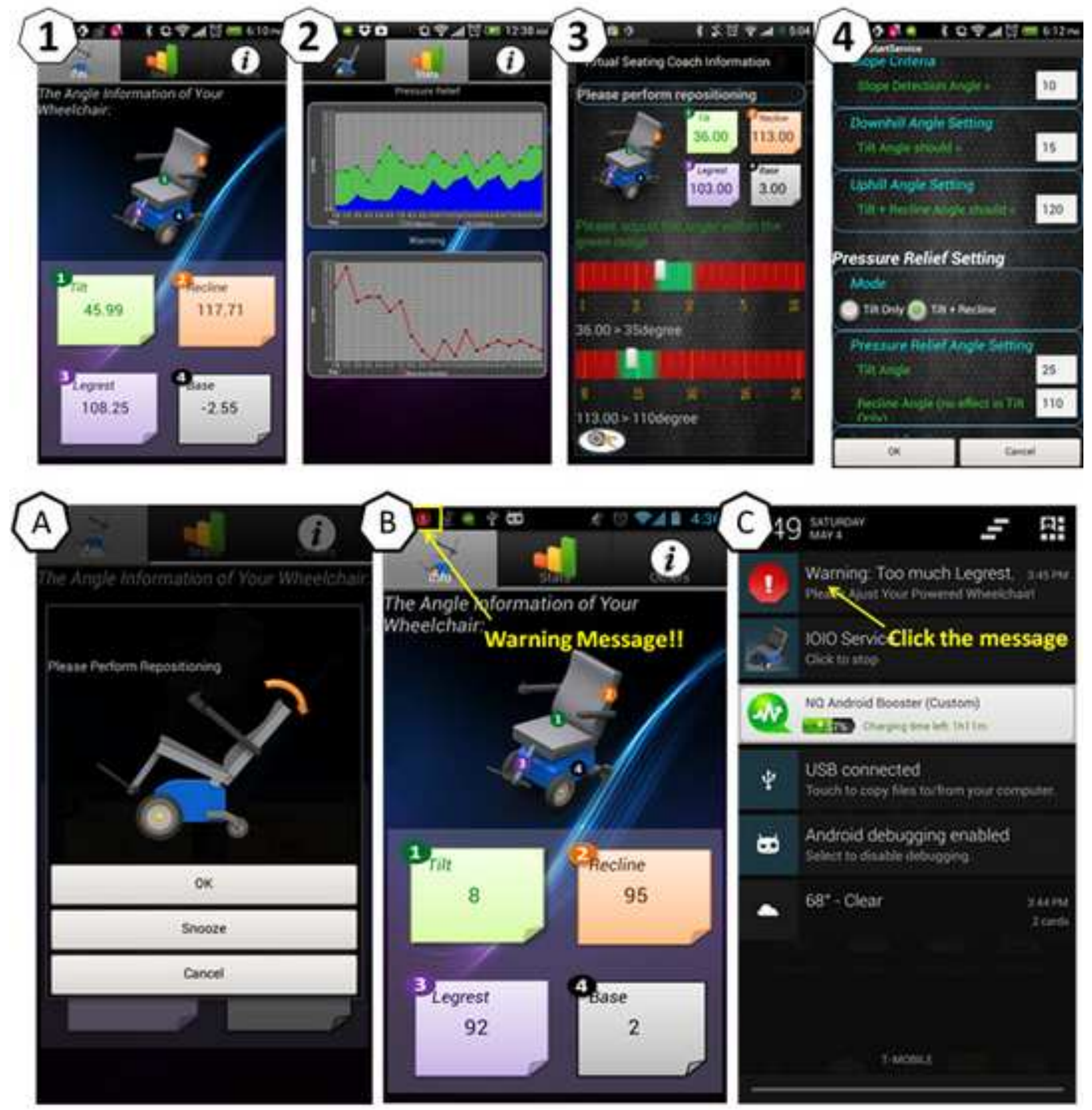


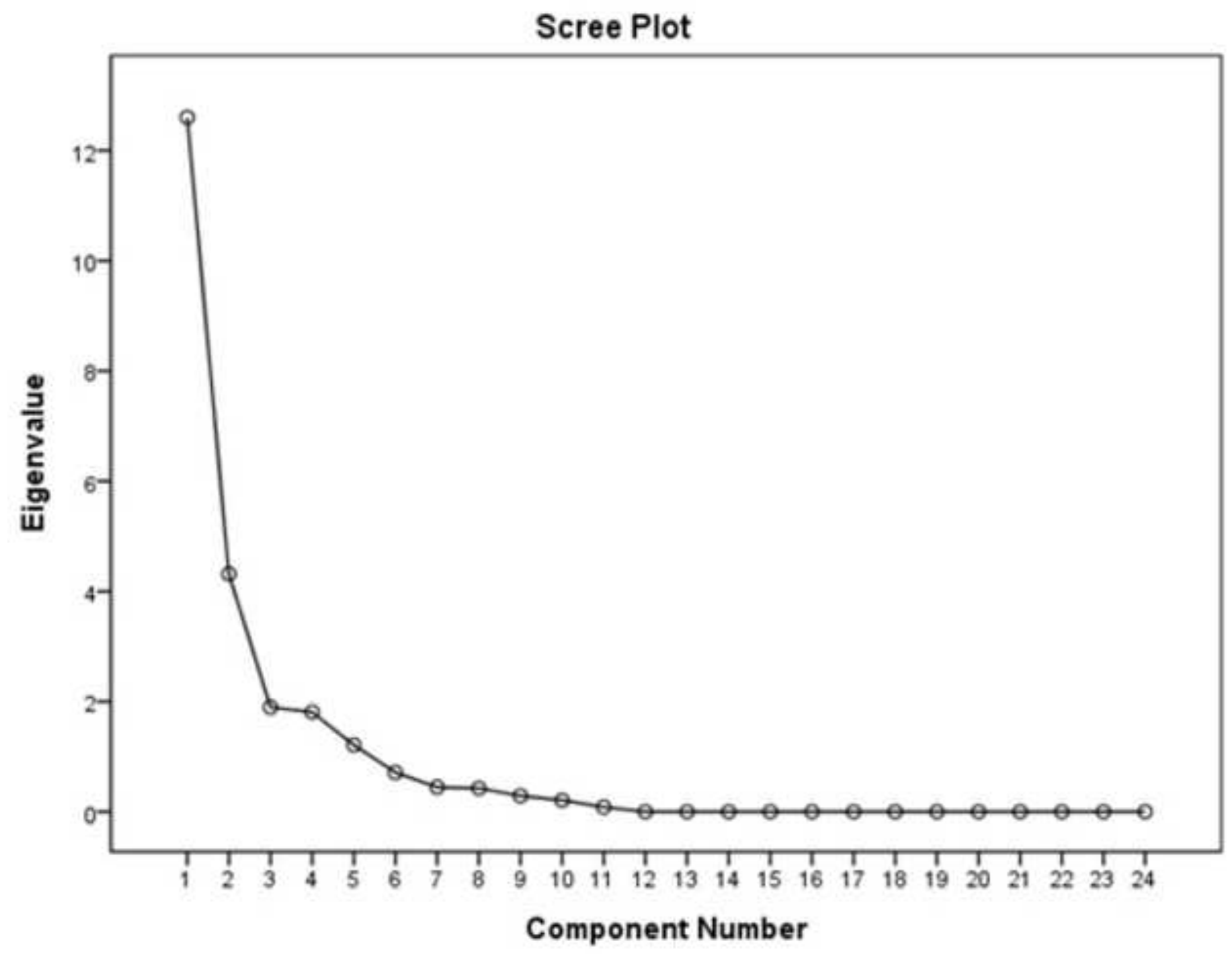




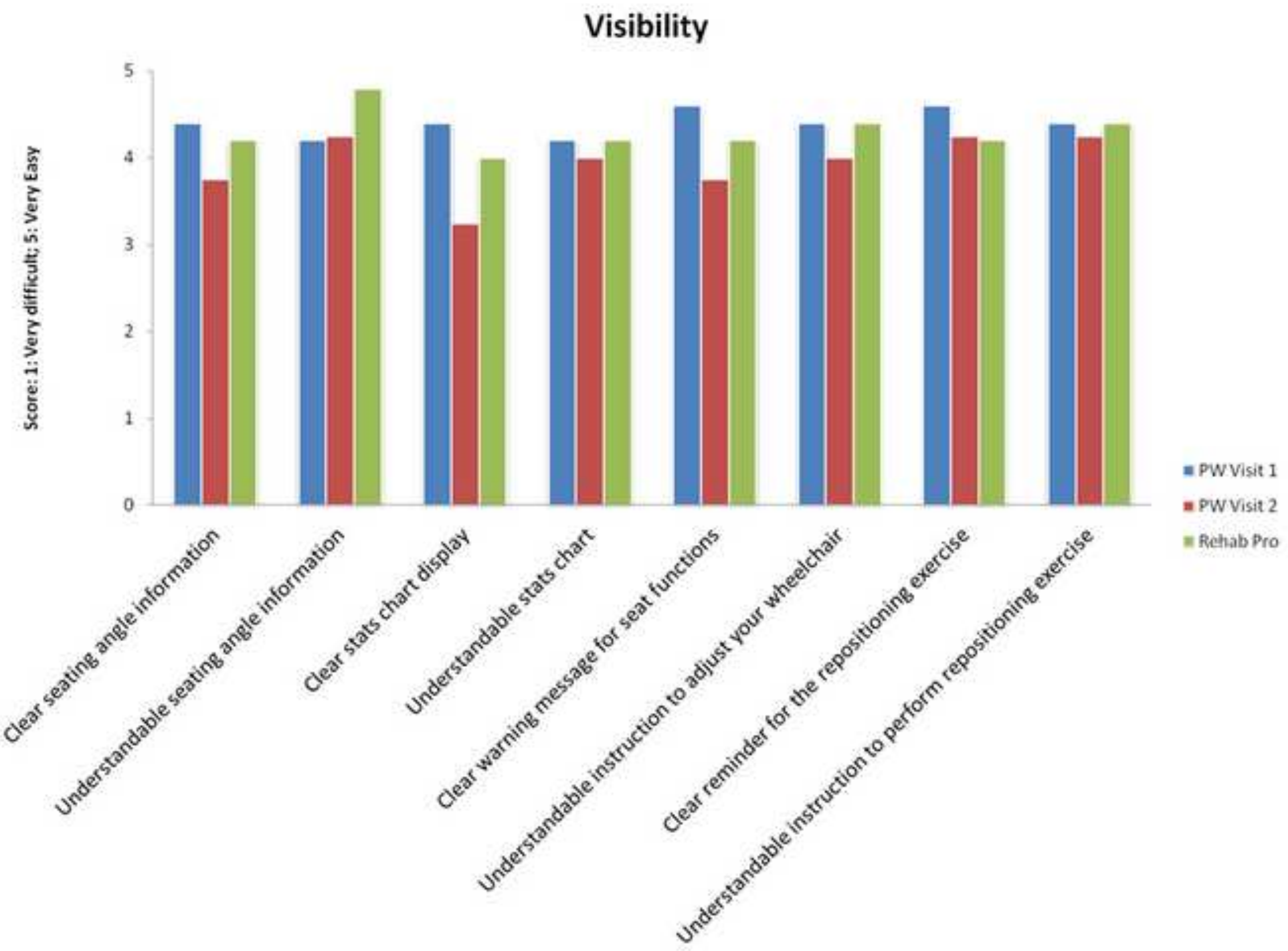




\section{Ease of use and Error prevention}

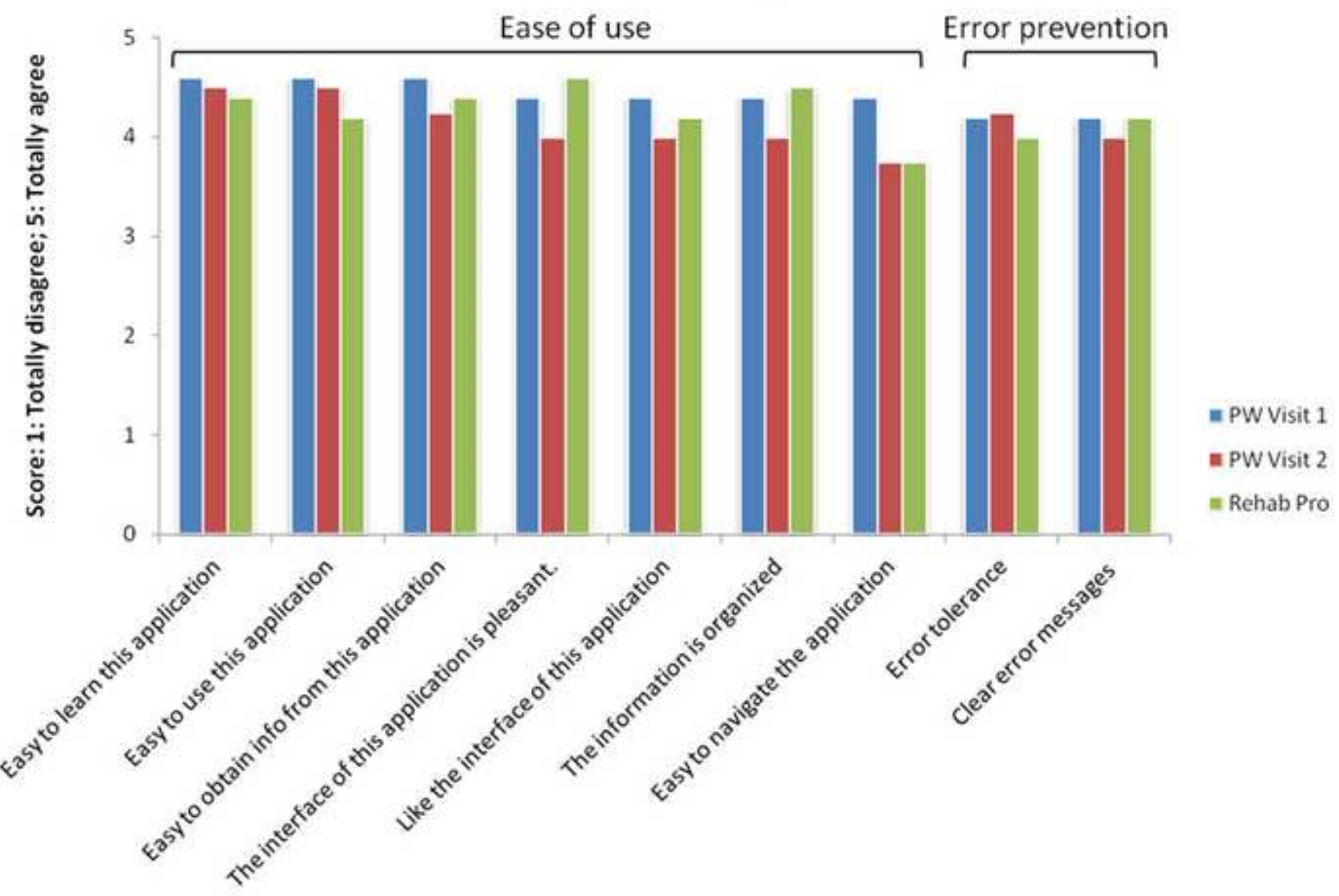




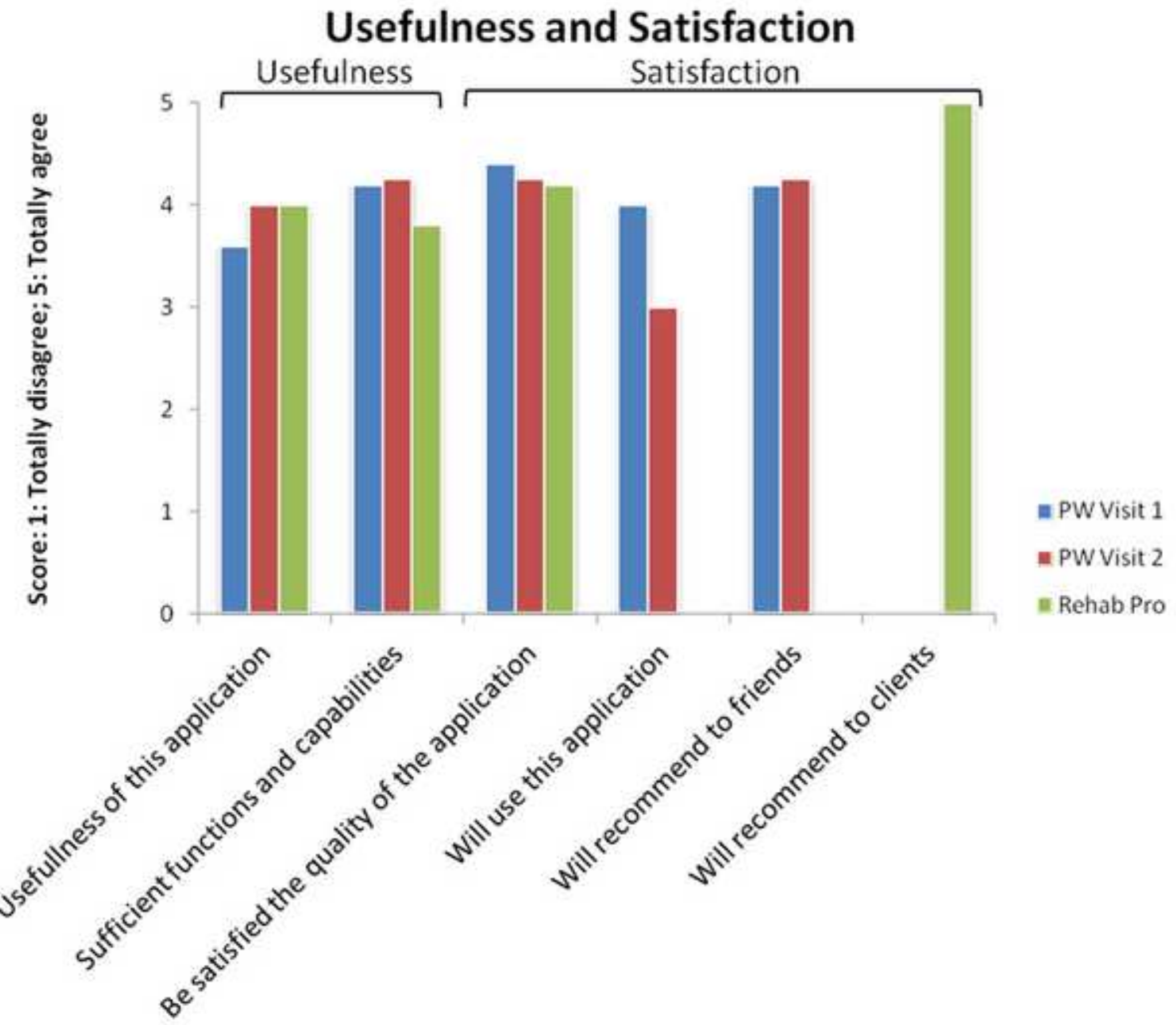


Table 1. The SVSC features that the participants liked (Question 2). ${ }^{a}$ The coaching instruction is shown in Figure 1-3. ${ }^{\mathrm{b}}$ The repositioning exercise reminder is shown in Figure 1-2. ${ }^{\mathrm{c}}$ One rehabilitation likes the color of the whole smartphone app.

\section{PW Visit $1 \quad$ PW Visit 2 Rehab Pro}

- The seating angle information

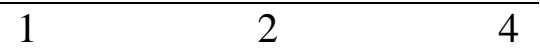

showed on the application

- $\quad$ Brief summary about the

1

1

2

repositioning performance and the number of safety warnings

- The coaching instruction for repositioning and safety warning ${ }^{\text {a }}$

- The automatic repositioning exercise

5

3

3 reminder $^{\mathrm{b}}$

- Customized setting for clients (If you

0

0

5 are a clinician)

- Other 
Table 2. The issues which are concerned by the participants (Question 3). ${ }^{a}$ One rehabilitation professional suggest the whole system should have a dashboard for them to review the PSFs usage of wheelchair users.

\section{PW Visit 1 PW Visit 2 Rehab Pro}

- The small display of a smartphone

0

0

3

might not show the information

clearly

- The location for the smartphone

5

4

2

cradle might interfere with transfers or other daily activities

- The wires connecting the

4

3

1

accelerometers might interfere with

transfers or other daily activities

- The accelerometers might be

1

0

1 damaged for long time usage

- Other

0

0

$1^{\mathrm{a}}$ 